

\section{REVISTA \\ TEORÍA Y PRÁCTICA \\ DE LA \\ ARQUEOLOGÍA HISTÓRICA LATINOAMERICANA}

ISSN: 2250-866X (impreso) | ISSN: 2591-2801 (en línea)

AÑO VIII, VOLUMEN 9, PRIMAVERA DE 2019

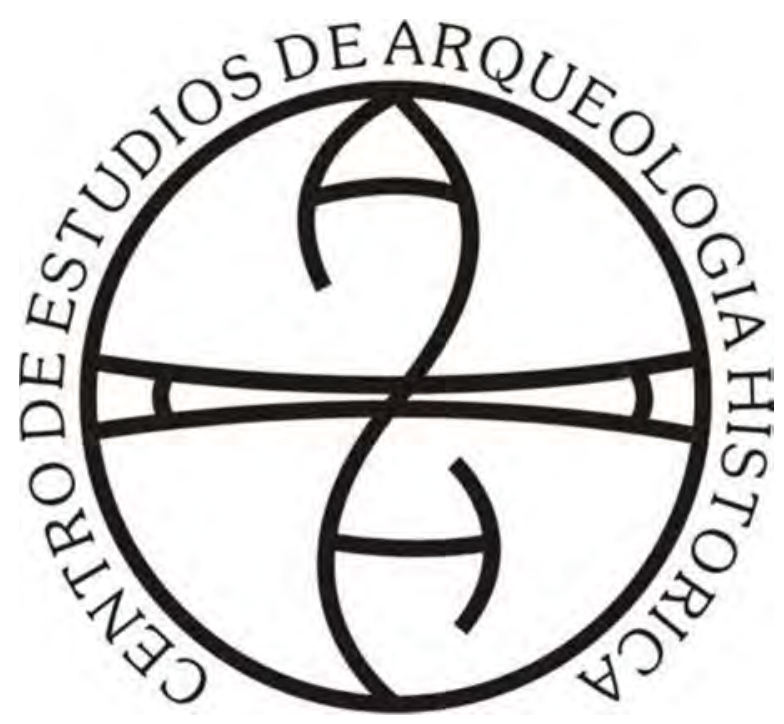

CENTRO de Estudios de ARqueOlOGía HistóricA

FACULTAD DE HUMANIDADES Y ARTES | UNIVERSIDAD NACIONAL DE ROSARIO 
(Universidad Nacional de Rosario, Universidad Nacional de Río Cuarto,

Universidad Nacional de San Juan, Universidad de la República, Universidad Nacional de Trujillo)

\author{
AUTORIDADES DE LA UNIVERSIDAD NACIONAL DE ROSARIO \\ RECTOR: Lic. Franco Bartolacci \\ VICE-RECTOR: Od. Darío Macía \\ SECRETARIO GENERAL: Prof. José Goity \\ SECRETARIA ACADÉMICO Y DE APRENDIZAJE: Dr. Marcelo Vedrovnik \\ SECRETARÍA DE CIENCIA TECNOLOGÍA E INNOVACIÓN \\ PARA EL DESARROLLO: Ing. Guillermo Montero.
}

\author{
AUTORIDADES DE LA FACULTAD DE HUMANIDADES Y ARTES \\ DECANO: Prof. Alejandro Vila \\ VICEDECANA: Prof. Marta Varela \\ SECRETARIA ACADÉMICA: Dra. Marcela Coria
}

\author{
AUTORIDADES DEL CENTRO DE ARQUEOLOGÍA HISTÓRICA \\ DIRECTORA: Dra. Ana Rocchietti \\ SECRETARIA: Prof. Nélida de Grandis \\ PROSECRETARIA: Lic. Marianela Bizcaldi
}

DIRECTORAS - EDITORAS:

Dra. Ana Rocchietti y Prof. Nélida De Grandis

SECRETARIA DE EDICIÓN: Dra. Irene Dosztal

Este número es co-edición de las ponencias

del VIII Congreso Nacional de Arqueología Histórica (2018) entre:

Centro de Estudios en Arqueología Histórica: Directora Ana Rocchietti

Centro de Estudios en Arqueología Regional: Director Fernando Oliva

Centro de Estudios en Arqueología Subacuática: Directora Mónica Valentini

Departamento de Arqueología, Escuela de Antropología: Director Fernando Oliva
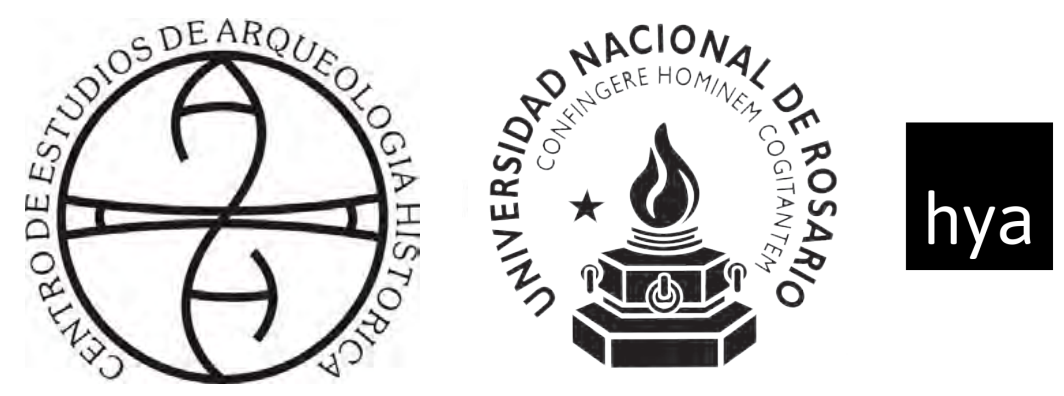

Facultad de Humanidades

y Artes_UNR
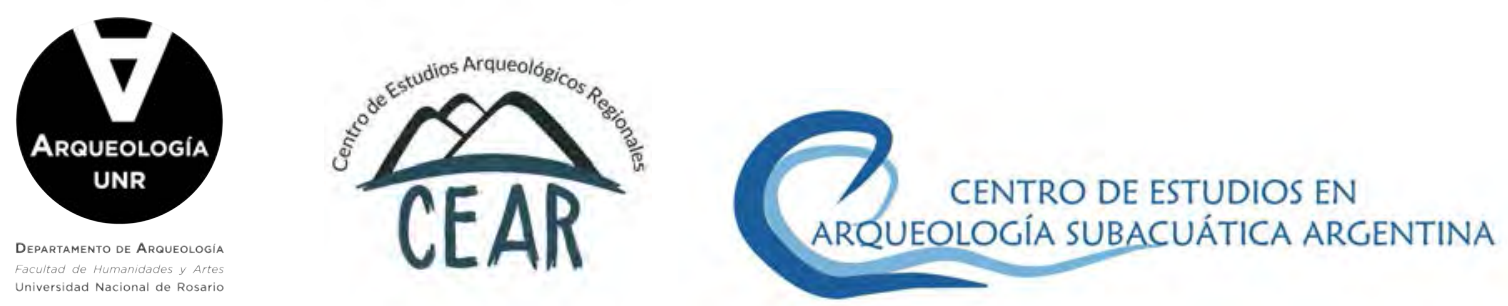
Comité Permanente de los Congresos Nacionales de Arqueología Histórica

Dr. Daniel Schávelzon (Universidad Nacional de

Buenos Aires)

Prof. María Teresa Carrara (Universidad Nacional de Rosario)

Prof. Carlos Baldassarre (Museo Municipal de Río

Grande, Tierra del Fuego) in memoriam

Dr. Mariano Ramos (Universidad Nacional de Luján, CONICET)

Dr. Horacio Chiavazza (Universidad Nacional de Cuyo)

Dra. Ana María Rocchietti (Universidad Nacional de

Rosario, Universidad Nacional de Río Cuarto)

Lic. Facundo Gómez Romero (Universidad Autónoma

de Barcelona)

\section{Comité Científico}

Dra. Tânia Andrade Lima (Universidade Federal do Rio de Janeiro)

Prof. Réginald Auger (CELAT/Département des

Sciences Historiques, Université Laval, Canadá)

Dr. Roberto Bárcena (Universidad Nacional de Cuyo, CONICET)

Dra. Marta Bonaudo (Universidad Nacional de Rosario, CONICET)

Dr. Leonel Cabrera (Universidad de la República, Uruguay)

Dr. Luis María Calvo (Universidad Católica de Santa

$\mathrm{Fe})$

Prof. Juan Castañeda Murga (Universidad Nacional de

Trujillo, Perú)

Dr. Carlos Ceruti (Museo de Ciencias Naturales y

Antropología "Prof. Antonio Serrano”. Paraná)

Dr. Horacio Chiavazza (Universidad Nacional de Cuyo)

Dra. Silvia Cornero (Universidad Nacional de Rosario)

Prof. Pedro Paulo Funari (Universidade Estadual de

Campinas, Brasil)

Lic. Jorge A. Gamboa Velásquez (Universidad Nacional

Santiago Antuñez de Mayolo, Perú)

Dr. Eduardo Alejandro García (Universidad Nacional de

San Juan, CONICET)

Prof. Nélida De Grandis (Universidad Nacional de

Rosario)

Dr. Juan Bautista Leoni (Universidad Nacional de

Rosario, CONICET)

Dra. Amancay Martínez (Universidad Nacional de San

Luis)

Dra. Catalina Teresa Michieli (Universidad Nacional de

San Juan, CONICET)

Lic. Fernando Oliva (Universidad Nacional de Rosario)
Ing. Adrián Pifferetti (Universidad Tecnológica Nacional Regional Rosario)

Dr. Mariano Ramos (Universidad Nacional de Luján, CONICET)

Dra. Ana María Rocchietti (Universidad Nacional de Rosario)

Dr. Daniel Schávelzon, (Universidad Nacional de Buenos Aires, CONICET)

Dra. Carlota Sempé (Universidad Nacional de La Plata)

Dr. Mario Silveira (Universidad Nacional de Buenos

Aires)

Dra. Silvia Simonassi (Universidad Nacional de Rosario)

Dra. Alicia Tapia (Universidad Nacional de Buenos Aires, Universidad Nacional de Luján)

Lic. Mónica P. Valentini (Universidad Nacional de Rosario)

Agrim. Benito Vicioso (Universidad Nacional de Rosario)

\section{Evaluaron este volumen}

Roberto Bárcena, María Teresa Boschin, Leonel Cabrera, Ulises Camino, María Rosa Carbonari, Carlos Ceruti, Horacio Chiavazza, Nicolás Ciarlo, Silvia Cornero, Eduardo Crivelli, Javier García Cano, Martín Gentinetta, María Laura Gili, Carlos Landa, Matilde Lanza, Melina Malandrino, Sebastián Pastor, Victoria Pedrotta, Josefina Piana, Mercedes Podestá, Mariano Ramos, Daniel Schavelzon, Diana Tamburini, Mónica Therrien, Mónica Valentini y María Teresa Boschin

\section{Diseño y diagramación}

Eugenia Reboiro

(eugenia.reboiro@gmail.com)

\section{Curadoría}

Ana Rocchietti e Irene Dosztal

Foto de tapa: Material arqueológico, del texto de Tapia et al.

\section{Propietario responsable:}

Facultad de Humanidades y Artes, Universidad Nacional de Rosario Centro de Estudios de Arqueología Histórica

Entre Ríos 758. Rosario, provincia de Santa

Fe (2000). Argentina.

Telf.: +54 (0341) 4802670

E-mail: ceahunr@gmail.com

Decreto Ley 6422/57 de Publicaciones

Periódicas 


\section{Índice}

Editorial

El origen del patrimonio histórico y su conservación integral en la contemporaneidad.

Yanina Aguilar

Prospección de basurales históricos de la ciudad de Rosario.

Soccorso Volpe y Gustavo Fernetti

Desconstrucción de un paisaje urbano. El Montevideo del extramuros colonial, aportes de la arqueología a su identidad actual. Ana Gamas

La Calota de Ameghino: reconsiderando un viejo tema desde la arqueología histórica.

Daniel Schávelzon

Análisis zooarqueológico de los restos hallados en una estructura sanitaria vinculada a momentos tempranos del actual barrio de Belgrano .71 Mario Silveira, Horacio Padula, Ricardo Orsini y Eva Bernat

La medida del tiempo: el reloj solar jesuita de La Cruz, provincia de Corrientes.

Fernando Oliva y María Cecilia Panizza

Sitio La Quinta: arqueología rural de campos serranos.

Flavio Ribero

Si no hay tabaco que no se note. Prácticas fumatorias en el fortin La Perra (1883-1885), La Pampa.

Alicia Tapia, Virginia Pineau y Melisa Ayelén Auge

El uso del tabaco y las pipas de caolin (clay pipes) en la frontera sur y oeste de la provincia de Buenos Aires en el siglo XIX.. .127

María del Carmen Langiano y Julio Fabián Merlo 
Patrimonio natural, urbano y arquitectónico de la costa en Mar del Plata. Federico Negroni

Huellas urbanas.

Ezequiel Serrot

Paisaje y patrimonio. La industria taninera en el siglo XX.

Provincia de Santa Fe.

Cristina Pasquali, Paola Milicic y Lara Ferré

Los desafíos de las arqueologías históricas latinoamericanas.

Andrés Zarankin

Sellos entre escombrales. Las lozas en el registro urbano

del Área Fundacional de Mendoza (siglo XIX y principios del siglo XX)

Lorena Puebla y Horacio Chiavazza

Evolución edilicia de la Plaza de Mayo de la Ciudad

de Buenos Aires (1580-1853).

Nicolás Ferrino

Gestión patrimonial en el rescate arqueológico y futuro Museo de Sitio

en Moreno 550, Ciudad Autónoma de Buenos Aires, Argentina.

María Eva Bernat, Ricardo Orsini, Horacio Padula y Mario Silvera 


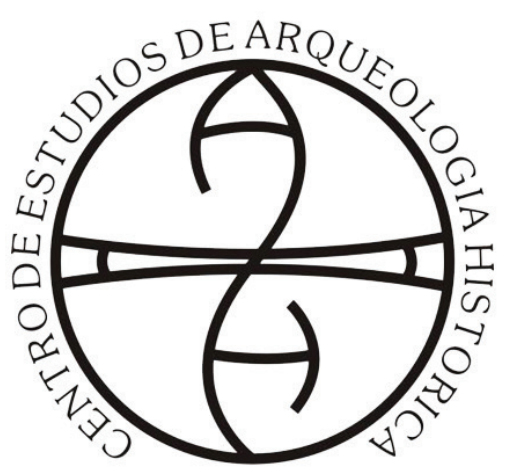

Centro de Estudios de Arqueología Histórica

Universidad Nacional de Rosario
Teoría y Práctica de la Arqueología Histórica

Latinoamericana | Año VIII. Vol. 9 | 2019

Revista del Centro de Estudios de Arqueología

Histórica, Facultad de Humanidades y Artes,

Universidad Nacional de Rosario

https://teoriaypracticaah.unr.edu.ar/index.php/index ceahunr@gmail.com

ISSN en línea: 2591-2801

ISSN versión impresa: 2250-866X

\title{
Evolución edilicia de la Plaza de Mayo de la Ciudad de Buenos Aires (1580-1853)
}

\author{
Nicolás Ferrino*
}

\begin{abstract}
Resumen
Este artículo se propone recorrer la evolución de la Plaza de Mayo, comenzando por una introducción extensiva a las ciudades hispanoamericanas, para luego volcarse de lleno en la Ciudad de Buenos Aires. Enumerando la serie de acontecimientos históricos y arquitectónicos que a modo de capas fueron superponiéndose, dejando algunas de estas, huellas que perduran hasta la actualidad, mientras otras desaparecen por completo.

Se podrá verificar entonces que en su mayor parte, el valor patrimonial de este sitio no esta materializado solo en forma de arquitectura construida, sino que en muchos casos, está presente por las consecuencias provocadas por el pasado demolido.

Será necesario relacionar la evolución de lo material con la realidad política, social y económica de su tiempo para, con ésta visión abarcativa, poder comprender mejor sus características y fundamentos.

Por último y a modo de conclusión, se pondrá en valor la actualidad de la Plaza de Mayo proponiendo una visión a futuro.
\end{abstract}

Palabras clave: Buenos Aires, ciudad, historia, patrimonio, arquitectura, urbanismo

\begin{abstract}
This article intends to explore the evolution of the Plaza de Mayo, starting with an extensive introduction to the Latin American cities, and then turning fully into the City of Buenos Aires. Enumerating the series of historical and architectural events that by way of layers were overlapping, leaving some of these, traces that last until today, while others disappear completely.

It can then be verified that for the most part, the heritage value of this site is not materialized only in the form of a built architecture, but in many cases, it is present due to the consequences caused by the demolished past.
\end{abstract}

\footnotetext{
* Facultad de Arquitectura, Diseño y Urbanismo. Universidad de Buenos Aires. ferrino.arq@gmail.com
} 
It will be necessary to relate the evolution of the material with the political, social and economic reality of its time in order to, with this comprehensive vision, be able to better understand its characteristics and foundations.

Finally and by way of conclusion, the current situation of the Plaza de Mayo will be valued, proposing a vision for the future.

Keywords: Buenos Aires, city, history, heritage, architecture, urbanism

\section{Introducción}

El sábado 11 de junio, con harta ceremonia, funda Garay Buenos Aires, en el nombre del Padre, del Hijo y del Espíritu Santo. Esta armado como para un torneo y en su coraza fulgura el sol. Dijérase un caballero andante, un Galaor, un Amadís de Gaula, mientras recorre el descampado, alrededor del árbol de justicia que acaban de erigir. De acuerdo con el rito antiguo, desnuda la espada, corta hierbas y tira unos mandobles terribles, hacia el norte, hacia el sur, hacia el este y hacia el oeste.

A su vera aguardan los setenta hombres, algunos de pie y otros de hinojos, con atavios de fiesta, y entre ellos, henchida la falda crujiente sobre la cual reposan sus manos ásperas, Ana Díaz, la única mujer.

Juan de Garay, tremebundo como un enorme crustáceo de plata, bracea penosamente, mientras repite las formulas de la toma de posesión. Alza la visera del yelmo, mira hacia el río triste y hacia el cielo de nubes quietas y sus ojos descansan en Ana, que esta rezando por lo bajo.

Y Ana sonríe. (Mújica Laínez, 1950)

Este fragmento de la "La Fundadora” de Mujíca Laínez da una idea casi romántica del ritual de la fundación de una ciudad en la América de fines del siglo XVI, y aunque breve, es contundente y muy grafico el relato de lo que pueden haber sido aquellos primeros instantes de la ciudad de Buenos Aires, de la Plaza de Mayo.

Esa instancia en la que todavía era una idea, un proyecto, en la que no se veía más que el descampado, el cielo, el río y su ribera barrosa e inestable y los setenta hombres, se ponía en marcha el proceso de transformación de esa realidad desoladora para los venidos de Europa, que actualmente es una urbe de 3 millones de almas.

\section{Génesis de las Plazas Hispanoamericanas}

La plaza en la América colonial, surge como elemento estructurante de la nueva urbe planteada en las Leyes de Indias.

Legislación especial iniciada en la época de Carlos V, rigió la fundación de las ciudades hispano-americanas, determinando la situación, trazado y rango que debían tener. Dicha legislación está contenida en el libro IV, titulo siete de las Leyes de Indias: De la población de las ciudades, villas y pueblos. (Solá, 1935)

En esta suerte de compendio de normativas para la colonización de las Indias, no solo está reglamentada la materialización de los asentamientos, sino también la forma de proceder de cada uno de los actores que intervenían en la administración del territorio, la participación de las distintas órdenes religiosas que evangelizaban al indígena así como todo lo referente al comercio entre otros temas. 


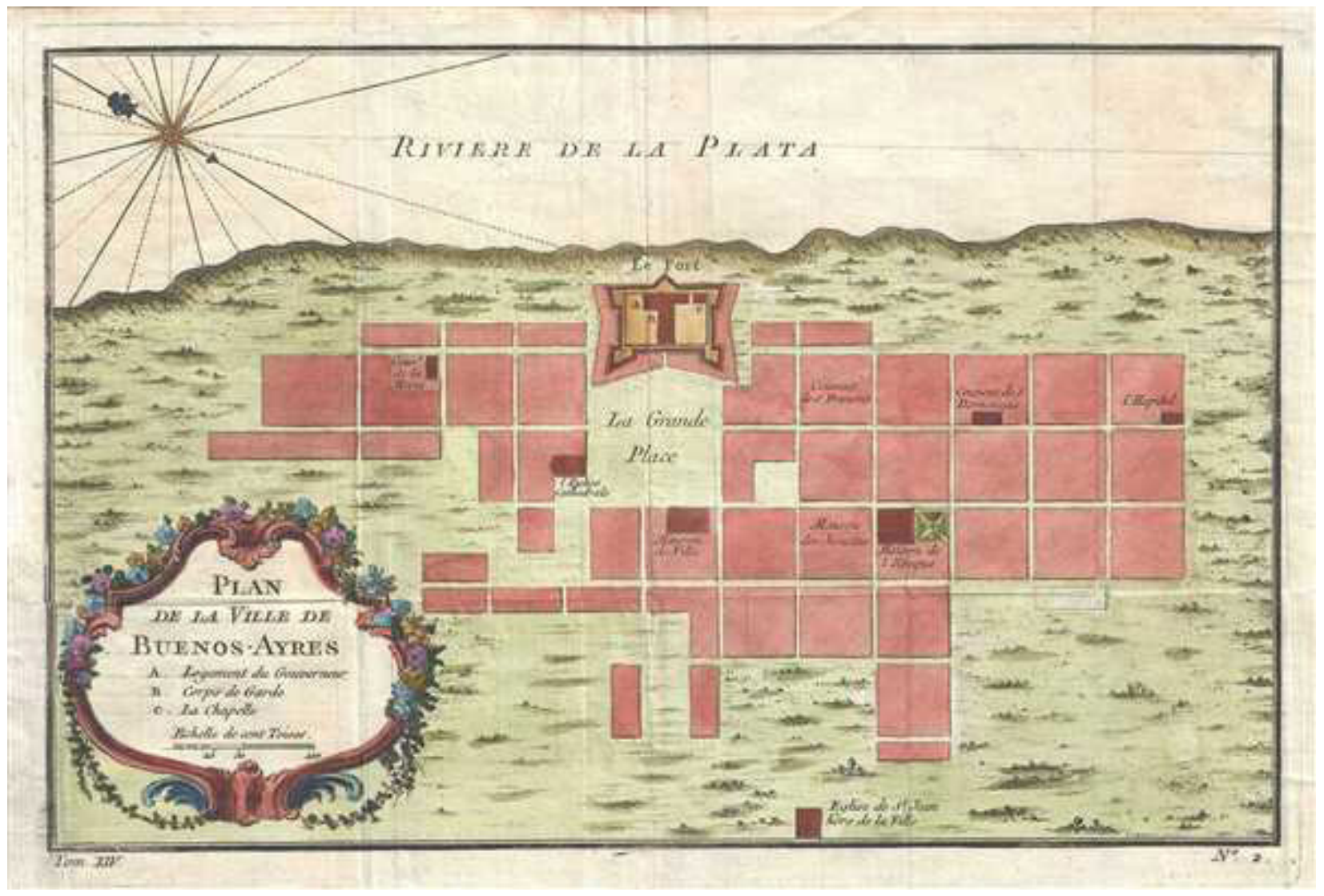

Figura I. Plan de la Ville de Buenos Ayres, París, 1749, BELLIN, J. N. Colección Ferrino.

La plaza es el tema central de este texto y para esta, la legislación era muy clara, aunque no fue rigurosa su implementación en los diversos casos que encontramos en Latinoamérica. Podemos comenzar mencionando que desde este espacio al principio imaginario, comienzan a desplegarse las calles y cuadras a través del territorio, conformando la cuadrícula.

Este sistema de calles que se cruzan en forma ortogonal, a distancias una de otra, de alrededor de 100 varas, es producto de la necesidad de crear asentamientos ex-novo con la capacidad de extenderse y ampliarse de manera ordenada, en paralelo con el posible crecimiento demográfico.

Sin intenciones de ahondar demasiado en el origen de este tipo de ciudad, sólo me remitiré a aclarar que el nacimiento de la cuadricula como estructurante de un tejido urbano, nace en Grecia, más precisamente en Mileto, y luego es adoptado por los romanos con algunas diferencias, ya que en el ejemplo griego la medida de la cuadricula es variable, y se adapta a las situación topográfica en las que se inserta. Para las ciudades romanas la distancia entre calles es invariable.

Cabe destacar que la reutilización de este sistema en la colonización del territorio americano luego de 1400 años, se debe en parte a la mención del mismo en los escritos de Vitruvio, profusamente estudiados y difundidos a partir del Renacimiento. Por otra parte, el método romano como referente, está íntimamente ligado al origen de gran cantidad de ciudades hispanas que nacieron como ciudades del imperio, tal es el caso de León y Lugo, o la deshabitada Itálica, donde la presencia del cardo y el decumano y los trazados regulares fueron generatrices del tejido. 
Resulta muy interesante contrastar el capítulo en el que Vitruvio se refiere al Foro (Libro V, Cap. I), con lo que se dictamina para la conformación de la plaza planteada en la ley IX del libro IV de las Leyes de Indias:

En las ciudades de Italia no es posible proceder de la misma manera porque desde nuestros antepasados se nos ha transmitido la costumbre de celebrar los juegos de gladiadores en la plaza pública. Así pues, para comodidad de los espectadores es preciso hacer más espaciosos los intercolumnios, intercalar bajo los pórticos y en todo el contorno tiendas de cambistas, y hacer en los entablados superiores, estancias que sirvan para el tráfico y para comodidad del público. La superficie de estas plazas públicas debe estar en proporción con la densidad de la población, de modo que ni resulten insuficientes por su capacidad, dadas las necesidades, ni parezcan demasiado desiertas debido al escaso número de concurrentes. Su anchura se podría determinar muy bien dividiendo la longitud en tres partes y dando dos a la anchura; de este modo su forma será oblonga, y su disposición apropiada a las exigencias de los espectáculos y a la comodidad de los espectadores. (Vitruvio, 1997)

La plaza mayor donde se ha de comenzar la población, siendo en costa de mar, se debe hacer al desembarcadero del puerto, y si fuere lugar mediterráneo, en medio de la población; su forma en cuadrado prolongado, que por lo menos tenga de largo una vez y media su ancho, porque será más a propósito para las fiestas de a caballo y otras; su grandeza proporcionada al número de vecinos, y teniendo en consideración a que las poblaciones puedan ir en aumento, no sea menos que de doscientos pies de ancho y trescientos de largo, ni mayor de ochocientos pies de largo y quinientos treinta de ancho, y quedará de medida y buena proporción, si fuera de seiscientos pies de largo y cuatrocientos de ancho. (Solá, 1935)

Las similitudes son evidentes sobre todo en lo que respecta al tamaño y a las proporciones, aunque también se puede hacer una relación en lo referente a la función del espacio, ya que ambos mencionan la posibilidad de realizar fiestas y eventos. Pero en lo relacionado a las medidas y las proporciones de ésta, es preciso realizar una lectura más detenida para comprender que con distintos conceptos o formas de expresarlo, el espacio al que se tiende en ambos casos es el mismo, y esto es herencia del pasado romano de las primeras urbes hispánicas, donde el centro social, político y económico necesita una calidad espacial tal, que contenga a los ocupantes, entendiendo por plaza contenedora a aquella que por sus proporciones permite el desarrollo de las actividades urbanas al aire libre sin resultar en exceso espaciosa. Estas medidas estaban implícitas en la memoria de la población urbana de las ciudades nacidas en el seno del imperio romano, por herencia también en los habitantes de las urbes hispánicas, así como hoy en los latinoamericanos que residimos en los productos urbanos de la conquista española de las Indias.

Aunque la legislación dada en estas leyes era muy clara, son muy pocos los casos en los que ésta se respetó a rajatabla ya que las condiciones en las que se establecían las ciudades hacían en muchos casos difícil la aplicación de las mismas. Es el caso de Cuzco, implantada sobre la capital del Imperio Incaico, de trazado irregular y con una plaza central de gran extensión que para el habitante hispano resultaría “desierta” e incómoda. Para este caso, se dividió el gran espacio en dos con un sector en el medio que se construyó y que dio como resultado una plaza principal y una secundaria (la plaza de armas).

De cualquier forma el conquistador siempre tendió a generar espacios propicios para albergar las actividades antes mencionadas, de manera tal que los ocupantes se sintieran contenidos en un ámbito que resultara de alguna manera familiar a lo que por vivencia consideraban un entorno urbano. 
Teoría y Práctica de la Arqueología Histórica Latinoamericana | Año VIII. Vol. 9 | 2019

ISSN en línea: 2591-2801 | ISSN versión impresa: 2250-866X

\section{La frontera sur}

Para comenzar es necesario detenerse en la condición topográfica sobre la cual se implanta la Ciudad de Buenos Aires y más específicamente la Plaza de Mayo. Estamos refiriéndonos a la ribera del Río de la Plata, a la que llega la infinitud de la Pampa. En este caso la situación es muy particular, ya que se trata de una vasta llanura que remata en una abrupta barranca que cae hacia el río de lecho barroso y de muy poca pendiente donde la línea que divide el agua de la tierra puede variar cientos de metros en el transcurso de un día.

Aunque parezca por ésta condición geográfica arbitraria la ubicación de un asentamiento ex-novo, Buenos Aires no fue emplazada en un lugar elegido al azar. Para la fundación se seleccionó un sitio siguiendo las recomendaciones de las Leyes de Indias en su ley primera según transcribe (Solá, 1935) "que en la costa del mar sea el sitio levantado sano y fuerte, teniendo consideración al abrigo, fondo y defensa del puerto, y si fuera posible no tenga el mar a mediodía, ni poniente”

La observación de un plano primitivo de la ciudad de Buenos Aires, permite ver que, en lo que a topografía respecta, aparecen tres elementos fundamentales: el Río de la Plata, el Riachuelo y por último la barranca.

Ésta no aparece como un desnivel paralelo a la costa propiamente dicha, sino que es una suerte de explanada unos metros más alta que la media del área que al avanzar sobre el río genera una bajada de pendiente bastante abrupta que garantiza un emplazamiento consolidado frente a la inestabilidad del barro y los caprichos de la marea rioplatense.

No es casual entonces que la plaza (como centro de la urbe) en conjunto con el fuerte, aparezcan sobre el borde de esta barranca en el punto donde ésta se encuentra más cerca de la línea promedio de las aguas del río, y a su vez a una distancia prudencial del sitio elegido para puerto en lo que hoy es la bajada del parque Lezama, hasta la que llegaban las aguas de la desembocadura del Riachuelo y donde las embarcaciones podían fondear con cierta seguridad.

Esa lengua de tierra más alta que se acerca al río desde la llanura, separa los terrenos inestables y barrosos a merced de las variaciones del curso de agua, de los altos y consolidados, indispensables para la salud de los habitantes y de las construcciones. Este fue el emplazamiento que a Juan de Garay le resultó más apropiado para fundar la ciudad, delimitar la plaza mayor, instalar el fuerte para defenderla y bien conectado con el lugar propicio para la instalación del puerto, que en definitiva fue la razón primera de la expedición fundadora.

Cabe aclarar que ése instante relatado por Mujica Láinez no es el solo el principio de la ciudad actual, sino que también es el final de una empresa que había comenzado más de 40 años atrás cuando Pedro de Mendoza decide seguir los pasos de Solís, quien había descubierto este formidable curso de agua, para establecer una salida alternativa a las cargas provenientes del Alto Perú sin tener la necesidad de rodear el continente por los peligrosos pasos australes. Empresa que fracasa rotundamente con el intento de consolidar el primer fuerte de Santa María de los Buenos Aires de 1536 y que a su vez trae consigo la fundación de otros tres asentamientos remontando el Plata y luego el Paraná como son el Fuerte de Santo Espíritu, Santa Fe y Asunción.

Desde la fundación hasta bien entrado el siglo XVIII, Buenos Aires fue una pequeña ciudad de arquitectura efímera, tanto la civil como la institucional. La Plaza de Mayo no era más que un gran escenario de tierra en la que se celebraban las fiestas, ejecuciones y velorios. Un espacio delimitado a regla bajo la legislación vigente para el resto de las ciudades, pero que en la práctica carecía de todo elemento que remitiera a la plaza hispana que los habitantes tenían como modelo. No había recova, ni una iglesia que 
se pudiera jactar de ser catedral, ni edificio institucional, ni siquiera una fortificación defensiva digna de un puerto. Sin embargo, aunque la plaza fuera imaginaria en lo material, después de 150 años de vida ya se había consolidado como corazón de la pequeña aldea. En primera instancia, sólo había un rectángulo simbólico sobre un plano de tierra dentro del cual por ley no se podía construir, que fue materializado como plaza principal por su función, por las actividades que se llevaban a cabo, por los individuos que hicieron de este espacio imaginario su lugar para relacionarse, comerciar, etc., y que lentamente fue consolidándose por la arquitectura que empezó a generar bordes.

Podemos decir entonces que, primero fue plaza por uso sobre un plano, y que paulatinamente se materializó espacialmente por la construcción del perímetro arquitectónico.

Con la llegada del siglo XVIII comenzó una etapa de crecimiento de la mano de las obras civiles y militares, y especialmente las religiosas que sentaron precedente en cuanto a la imagen corbusierana del perfil desde el río. A la mirada de los viajeros que arribaban a estas tierras la todavía incipiente ciudad-puerto se mostraba como una aldea baja de la que sólo sobresalían las cúpulas y torres de templos de las diferentes órdenes que se instalaron. Ya para 1750 se divisaban en el perfil las siluetas correspondientes a la iglesia jesuita de San Ignacio, la de La Merced, Santo Domingo, Santa Catalina de Siena, San Francisco y por supuesto la Catedral; Cabe aclarar que la torre del Cabildo no se construiría hasta la primera década del siglo XIX.

Buenos Aires generaba desde lo lejos una ilusión que se desvanecía, según relatos de mediados del XVIII, al adentrarse en el Río de la Plata. Comenzaba a divisarse el perfil de Buenos Aires que emergía de la vasta llanura que dividía las aguas del cielo. Las torres y cúpulas que se recortaban en el horizonte hacían pensar en una urbe de gran desarrollo. Al desembarcar no invadía más que la desilusión ya que la ciudad poseía una construcción precaria ordenada sobre la base de una cuadrícula ortogonal de calles de tierra que ante la lluvia se tornaban intransitables. A pesar de esto la arquitectura religiosa estaba bastante más desarrollada. Aún sin concluir las principales iglesias ya tenían en sus interiores así como en sus fachadas la intervención de los padres constructores jesuitas que embellecieron el paisaje urbano en la frontera sur de las indias con exponentes renacentistas o barrocos dependiendo de su origen. Andrés Blanqui (Andrea Bianchi), Juan Bautista Prímoli o Krauss por citar algunos, traían consigo la experiencia constructora y si bien ninguno era arquitecto, cada uno proyectaba sobre la base de la arquitectura predominante en su lugar de origen. Estos jesuitas constructores viajaban de las misiones a Córdoba o Buenos Aires llamados para intervenir en cuanto edificio de cierta complejidad los requiriera ya que por aquellos años en estas tierras no había arquitectos ni constructores con experiencia.

Durante la primera mitad del siglo XVIII el paisaje que circundaba la plaza cambió drásticamente. Esto se debe a dos razones fundamentales: en primer lugar, Buenos Aires ya no era una gobernación, había adquirido rango de capital de Virreinato, hecho que trajo un fuerte impulso a la construcción pública y religiosa. Hacia el Sudeste, el Cabildo aparecía como un edificio jerárquico dentro de la arquitectura de la ciudad. Surge de la necesidad de una sede para la justicia y la cárcel, encargándose en la primera década del 1700, según documentos del Archivo de Indias, el padre Prímoli de proyectar la planta y el ingeniero militar Petrarca de presupuestar y levantar el edificio. Esta obra introduce un elemento que luego será característico de la plaza, la recova, que se materializaba en el frente de la flamante construcción con una sucesión de arcos de medio punto apoyados en pilares.

Si bien esta galería perimetral de la plaza formaba parte de la legislación impuesta por las Leyes de Indias, no había aparecido hasta ese momento porque la modestia de la arquitectura incluso en el espacio central y principal de la ciudad era tal que el hecho de generar una cubierta apoyada sobre pilares que diera techo a los laterales era impensado. Incluso la Iglesia Catedral que se encontraba sobre la esquina 
Teoría y Práctica de la Arqueología Histórica Latinoamericana | Año VIII. Vol. 9 | 2019

ISSN en línea: 2591-2801 | ISSN versión impresa: 2250-866X

este carecía de una escala y jerarquía digna de una capital de Virreinato. Esta atravesaría gran cantidad de inconvenientes en lo constructivo siendo demolida y reconstruida en varias ocasiones quedando sin fachada hasta las primeras décadas del siglo XIX.

Volviendo a la arquitectura publica, hacia la barranca del río aparecía el fuerte de la ciudad, bastión defensivo típico de cualquier puerto español en las colonias. Técnicamente este conjunto de edificios militares y administrativos bordeado por una muralla, estaba muy lejos de los ejemplos que podemos encontrar en La Habana (Cuba) o en San Juan (Puerto Rico). Esto se debe, en parte, a que la ciudad no tenía la jerarquía e importancia de aquellas. Por otra parte Buenos Aires contaba con una defensa natural dada por la poca profundidad del río que sólo permitía el ingreso de embarcaciones de poco calado a través de un canal que pasaba por el frente del mismo fuerte, quedando así expuestas a una descarga a poca distancia de la artillería de defensa. Esta característica natural del acceso a la costa de Buenos Aires fue la que impidió en ambas invasiones inglesas la toma permanente de la capital del Virreinato ya que la fuerza invasora tuvo que desembarcar varios kilómetros al sur de la plaza y acercarse a pie, lo que le dio a los defensores tiempo de planificar y organizar una estrategia de retirada hacia la zona norte para luego retomar la ciudad. Cabe aclarar que en ninguno de los casos el fuerte ofreció una real resistencia, quedando en evidencia la poca utilidad que éste tenía en el cumplimiento de sus funciones. Este "Castillo" de Buenos Aires, como se lo denomina en algunos planos de la ciudad del siglo XVIII funciona desde la fundación de Buenos Aires como sede del gobierno, hecho que permaneció invariable hasta nuestros días ya que en ese mismo emplazamiento se encuentra hoy la Casa de Gobierno, hallándose el acceso de ésta en el mismo lugar en el que se hallaba el arco de triunfo (muy austero por cierto) a través del cual se accedía al fuerte.

Aunque su función defensiva era muy pobre, puede afirmarse que este elemento generaba un cierre espacial hacia el río, y este edificio que se mostraba hacia la plaza en forma de muro de ladrillo y barro con un acceso, era el único que jerarquizaba el eje de simetría longitudinal que sólo estaba acompañado por la presencia de la pirámide de mayo. Esta línea media que dividía a la plaza (y de algún modo a la ciudad) en norte y sur, a finales del XIX sería fuertemente reforzada por la apertura de la Avenida de Mayo al modo de las grandes avenidas que en la ciudad modelo de Paris unían nodos urbanos o edificios importantes.

Las viviendas privadas y comercios de una sola planta y construcción austera terminaban de cerrar materialmente este espacio. Si bien estas eran las más lujosas de la ciudad no se comparaban con las viviendas de dos pisos de los adinerados de Lima por citar un ejemplo, en las que además había un desarrollo estilístico acorde con el barroco, con balcones de madera y celosías.

\section{El lento desarrollo hasta el modelo agro exportador}

La arquitectura en la Argentina fue influenciada básicamente por dos corrientes:

La corriente norteña o de Humahuaca, relacionada con la construcción austera del noroeste argentino, se adaptaba a las condiciones sociales de la numerosa población indígena desarrollada culturalmente en el período prehispánico. Implantada generalmente en zonas desérticas como la puna y asociada con algunos aspectos de la arquitectura colonial del Perú y Bolivia.

Los templos católicos eran de pequeñas dimensiones, en la mayoría de los casos con techumbres en madera de cardón, con anchos muros de piedra o adobe que conformaban una nave única que remataba en un altar en cuyo retablo se concentraba toda la decoración. Estos eran generalmente productos de los talleres do Bolivia o Perú, especialistas en la talla de la madera y el dorado a la hoja. 
Teoría y Práctica de la Arqueología Histórica Latinoamericana | Año VIII. Vol. 9 | 2019

ISSN en línea: 2591-2801 | ISSN versión impresa: 2250-866X

Exteriormente estos templos modestos carecían casi por completo de decoración, que se limitaba al resalto de elementos que a falta de accesorios estilísticos, destacaban por su fuerte volumétrica. (Documentos de Arte Argentino, 1946)

Podemos agregar también que era usual la presencia del altar exterior generado por un acceso al edificio cubierto, con un balcón que se utilizaba para las prácticas religiosas.

Esta corriente, tuvo influencia en la zona noroeste del país, pudiéndose reconocer ejemplos arquitectónicos de la misma hasta en la actual provincia de Córdoba donde choca con la segunda corriente que influyó en la Argentina del período colonial, la del Atlántico.

La presencia de Buenos Aires como puerto y capital del Virreinato atrajo a constructores y artistas venidos directamente de Europa. Aunque esta ciudad no tenía escuela como las del Virreinato del Perú, funcionó como puerta de entrada de una serie de personajes que dejarían su marca como los padres Jesuitas antes citados. Estos trajeron consigo los modelos arquitectónicos italianos, alemanes o franceses que destacan por un respeto mucho mayor a las tendencias o modelos ya impuestos en el viejo continente. El modelo de templo de la contrarreforma, los órdenes clásicos etc., comenzaron a verse en la ciudad de Buenos Aires, la Provincia de Córdoba e incluso en las Misiones Jesuíticas donde estos elementos serían materializados por la mano de obra guaraní. Cabe destacar que en esta última experiencia aparecería una de las escuelas artísticas más importantes del territorio argentino, en la que los indígenas se hicieron diestros constructores, talladores o escultores, generando un estilo único, el arte Jesuítico-Guaraní.

La gran diferencia entre estas dos corrientes culturales, reside en que la condición de pureza de implementación constructiva, funcional o estilística de la edificación que aparece de la mano de los llegados por el puerto de Buenos Aires, está ligada a la ausencia de civilizaciones preexistentes con tradición constructiva desarrollada. Mientras que en el Alto Perú la cultura incaica proveyó mano de obra experimentada en la labranza de la piedra, la alfarería fina u otras actividades que generaron en todas las ramas del arte, un mestizaje que dio como resultado una escuela con características propias.

Es necesario conocer estos factores para comprender el porqué del contexto edilicio que en el año de 1810 vería al Virreinato del Río de la Plata convertirse en territorio independiente.

El Cabildo que había sido proyectado por el padre Prímoli y construido por el ingeniero militar Petrarca, era un edificio cuyo frente poseía el único tramo de recova hasta ese momento construida en la plaza, materializada por 22 arcadas, 11 en la planta baja e igual número en planta alta, con su eje de simetría y acceso jerarquizado por un conjunto a modo de arco de triunfo que el padre Blanqui había proyectado. Este modelo proveniente del renacimiento albertiano se transformó en una muletilla estilística que también aplicó en el frente de la catedral de Córdoba y en la Iglesia del Pilar. Como muchos otros constructores jesuitas, los ejemplos vistos en sus tierras natales eran copiados con leves modificaciones. Es curioso notar como esta fachada de arco triunfal fue aplicada casi de igual modo en un edificio público, en un templo de grandes proporciones como la iglesia cordobesa o en un edificio eclesiástico menor como la del Pilar en las, en ese entonces, afueras de la ciudad de Buenos Aires.

Esto es un claro ejemplo de la arquitectura importada en forma casi inalterada del viejo continente a través del puerto, y como éste podemos encontrar muchos ejemplos en las cercanías de la Plaza de Mayo, pero es interesante notar que en tiempos de la revolución de 1810 este paño central de la fachada del Cabildo se había reproducido en más de un edificio cercano a éste. Sin ir más lejos en la construcción lindera se encontraba la sede de la policía, mucho más modesta en sus dimensiones, que también tenía su acceso jerarquizado por este modelo. Al otro lado de la plaza el acceso al fuerte era un arco de triunfo absolutamente sintetizado a un bloque apenas articulado por una cornisa superior y una secundaria a la altura del arranque del arco. Esta construcción poseía toda la severidad característica de un emplazamiento militar 
ya que, con este elemento, se completaba la vinculación del interior del fuerte con la plaza a través de un puente levadizo que nunca se utilizó, con el que se atravesaba la pequeña fosa que bordeaba los muros.

En el año de 1803 se va a comenzar la edificación de la denominada Recova Vieja, que cambiará por completo la espacialidad de la plaza, dividiéndola en dos a la altura de las calles Defensa-Reconquista, por medio de dos alas con doce arcos cada una, unidas por uno de mayor envergadura en coincidencia con el eje del acceso del fuerte, y es justamente este último y principal el que estará jerarquizado por una reconstrucción casi virtual del modelo romano de puerta ceremonial.

Resulta interesante la comparación de estos cuatro ejemplos ya que todos toman el mismo motivo, pero materializado de diferente manera. El caso del Cabildo y la sede de la policía, poseen este elemento a modo de placa aplicada a un plano donde se desarrolla el tratamiento estilístico, éste podría ser el ejemplo que más se acerca al sistema albertiano. En el caso del acceso del fuerte, se elimina casi por completo la articulación en base al lenguaje clásico, pero lo que se reproduce es el modelo romano de arco triunfal en una analogía volumétrica. Por último la recova utiliza el plano articulado de igual manera que el cabildo y la sede de la policía pero éste, se aplicaba a una superficie menor. Sólo la estructura de la bóveda y los pilares se encuentran por detrás, por lo tanto las placas quedaban como un escenario teatral pudiéndose relacionar con algunas fachadas barrocas. Esto no es casual ya que el edificio en su totalidad funciona como una pantalla decorativa y volumétrica destinada a acotar espacialmente a la plaza, quedando ésta dividida en dos áreas que, como se explicó con anterioridad, eran más acordes a la proporción de superficie respecto de la poca contención que generaban los modestos bordes hasta ese momento construidos. Por otra parte las galerías de esta construcción rápidamente se poblaron de vendedores que a resguardo del sol y la lluvia, encontraron un ámbito más agradable para ejercer su actividad.

Con lo explicado anteriormente llegamos al lo que sería el entorno de los acontecimientos del 25 de mayo de 1810, una plaza dividida en dos por la recova, el edificio del cabildo con su flamante torre, la catedral aun sin fachada, y viviendas con comercios que ocupaban los restantes fondos. La plaza frente al fuerte, la de Armas, era más pequeña y de menor jerarquía, ésta todavía conservaba los restos del antiguo asentamiento jesuita (piquete de San Martín), siendo el trazado mucho menos definido ya que sus laterales poseían las dos grandes ochavas, deformando el rectángulo compositivo del modelo colonial.

La evolución edilicia de ambos sectores fue despareja, quedando relegada la de Armas respecto de la luego denominada 25 de Mayo, ya sea por su menor calidad espacial o su tamaño o, probablemente lo más importante, la jerarquía institucional de una en comparación con la otra.

El siglo XIX y la independencia trajeron consigo grandes cambios en todos los ámbitos, y por supuesto en lo edilicio. Siendo Buenos Aires protagonista de casi todos los conflictos dentro de la organización nacional, el corazón de la ciudad comenzó a evolucionar, lentamente hasta la mitad del siglo y luego de manera cada vez más acelerada y drástica.

El lado sur de la Plaza 25 de Mayo se consolidó en 1818 con la construcción de la Recova Nueva, que en sintonía con el lado este (Recova Vieja) y el oeste (Cabildo) continuaba con el proyecto de generar un borde sobre la base de galerías.

El primer signo de ruptura en lo arquitectónico con el pasado colonial viene de la mano de Bernardino Rivadavia, quien en 1821 decidió fomentar la construcción del frente de la catedral, que desde 1778 mostraba solo un muro de ladrillos con la huella de la bóveda de la nave principal y los cimientos de las torres de la antigua fachada demolida. Para esto Próspero Catelín, el arquitecto designado para la obra eligió como modelo el contrafrente del Palais Bourbon de Paris (actual Cámara de Diputados de Francia, edificado en 1722 por el arquitecto Giardini) en un claro giro estilístico. El neoclasicismo aparece en Europa de la mano de las ideas de la Ilustración que ya desde el siglo XVIII proponían el racionalismo 
en todos los ámbitos, el político, el social y artístico entre otros. Por esto la arquitectura clásica en su estado más puro vuelve a estar en boga siendo sometida a estudios con relación a este aspecto racional y matemático que la caracteriza. Rivadavia, en este momento ministro de la provincia de Buenos Aires, y con años de residencia en Europa viene empapado de estas ideas, y para la fachada de la Catedral de la Cuidad de Buenos Aires propone un frente templario que abraza el lado del edificio que da a la plaza con una columnata que genera una galería que trasciende el terreno de la iglesia del mismo modo que el cabildo para armar su recova. Esta intervención es interesante no sólo por la ruptura con la cultura colonial, sino también porque es una solución que aprovecha la legislación acerca de la recova que permite que se avance con la edificación sobre la superficie de vereda. El motivo de frente templario griego y las leyes de Indias encuentran en esta situación la posibilidad de combinarse generando un conjunto edilicio que se puede relacionar con la Madelaine de París, donde una cáscara a modo de templo períptero octástilo clásico envuelve un planteo basilical. En el caso de Buenos Aires el frente duodecástilo del arquitecto Catelín, envuelve el cuerpo en cruz latina con cúpula proyectado por Antonio Masella en 1754. Según Carlos E. Pellegrini, autor de numerosas pinturas de la Plaza de mayo en tiempos de Rosas, la catedral aún inconclusa es un elemento que da jerarquía al entorno de este espacio, pero critica el muy tímido basamento que no está a la escala de las dimensiones de la fachada. En efecto, observando los ejemplos clásicos o las adaptaciones parisinas, esta escalinata que sirve de base al conjunto tiene mayor dimensión en proporción con la totalidad, es decir, que al ejemplo porteño le faltan escalones. Esto no es por una imprecisión del proyectista sino que se debe, a que el templo original poseía poca diferencia de altura del piso respecto del nivel de suelo de la plaza. Esta obra tuvo un desarrollo muy lento y quedo concluida con los trabajos escultóricos en el tímpano a cargo de José Dubourfieu en 1863.

A partir de 1810 se inicia un período de luchas internas por la organización del estado entre las provincias y Buenos Aires.

El duelo entre dos concepciones políticas (el centralismo liberal y el federalismo) se insinuó desde poco después de la Revolución de Mayo y alcanzó su más profunda dramaticidad en 1820; La democracia doctrinaria sucumbió con la caída de Buenos Aires, y el triunfo de los caudillos trajo consigo la disgregación del país. (Romero, 1956)

Esta serie de acontecimientos dio como resultado la ausencia de la obra pública durante 40 años, pero la aparición de personajes con gran poder adquisitivo, generalmente grandes terratenientes o militares, generó obra privada y en la plaza esto se manifestó con la consolidación de viviendas como las construidas en 1818, los altos de Elorriaga, llamados así por tener dos plantas, que además continuaron con el proyecto fundacional de la recova. Estas no eran las casas de la familia sino que eran unidades de renta de pequeñas dimensiones que en muchos casos poseían el comercio en planta baja.

Si bien no eran ejemplos de gran calidad constructiva o refinamiento estilístico, significaron el cierre espacial definitivo del borde, que hasta ese momento no se había logrado. Es interesante mencionar una de las residencias más notables construidas en el periodo colonial, que todavía en las primeras décadas del siglo XIX seguía siendo una de las más atractivas, la casa de Domingo Basavilbaso, construida en 1782, que luego perteneció a los Azcuénaga y por último fue sede de la Aduana hasta que finalmente se la demolió en 1890. Estaba ubicada a pocas cuadras de la plaza, sobre la Av. Belgrano y destacaba por su decoración en la que resaltaba el acceso principal con un motivo barroco, posible referencia al frente de San Ignacio de tendencia alemana.

La ciudad que estaba creciendo, en este emplazamiento urbano evidenciaba la desaparición de la tapia y los techos de paja, que todavía eran denominador común en los alrededores, y que a pocas cuadras se convertían en zonas de terrenos de un cuarto de manzana con la residencia exenta. 
Teoría y Práctica de la Arqueología Histórica Latinoamericana | Año VIII. Vol. 9 | 2019

ISSN en línea: 2591-2801 | ISSN versión impresa: 2250-866X

El barrio de catedral al sur, residencial, se había conformado a partir del eje de defensa que comunicaba el centro con el puerto en parque Lezama. Esta calle de gran actividad en base al movimiento de gente hacia un puerto cada vez más activo encolumnaba a tres de las iglesias principales, San Francisco en el cruce con Alsina, Santo Domingo sobre la avenida Belgrano y la de San Telmo un poco mas alejada y sobre otro nodo de importancia urbana, la plaza del mercado de San Telmo, hoy Plaza Dorrego.

Hacia el norte el barrio inglés o de Catedral al Norte, de características más comerciales, también era atravesado por otro eje importante, la calle Florida, en ese momento empedrada ya que fue la primera completamente adoquinada y que ya desde ese momento era muy transitada por su gran cantidad de comercios. Este eje conectaba el centro con El Retiro, donde se llevaban a cabo las corridas en la Plaza de Toros que en 1809 dejó de funcionar para su posterior demolición. Pastor Obligado hace referencia a este tema en sus Tradiciones Argentinas:

Ocho años después (de 1809), bajo la dirección del maestro Sauces se demolía la plaza de toros, y sus ladrillos pasaron a formar los arcos del Retiro en el cuartel que desde 1817 se edificaba en su barranca. Al progresista gobierno del General Balcarce tocó expedir el decreto prohibiendo las corridas de toros. Algunos años siguieron lidiándose del otro lado de Barracas, con ocasión de alguna solemnidad, y en el interior del país por muchos más.

(Obligado, 1903)

En este sitio además se encontraba uno de los caserones más importantes de Buenos Aires, el del Gobernador Robles, que luego por la envergadura de la construcción pasaría a manos del ejército instalándose allí el ya desaparecido cuartel de artillería de Retiro.

Antes de continuar, es interesante detenerse en el texto de Obligado en lo referente a la reutilización de los ladrillos de la demolición de la plaza de toros. En 1817 la ciudad contaba con poca producción de ladrillos y no es casual que se demoliera este edificio en pos de la construcción de un cuartel militar, ya que el conflicto con las provincias estaba en uno de sus momentos más álgidos y la ciudad ya había adoptado la acción armada como forma de reprimir las sublevaciones al inestable gobierno central.

Hacia 1820 el país estaba fuertemente dividido en regiones gobernadas por caudillos que con la defensa de intereses locales y tradicionalistas conseguían el apoyo del pueblo que veía en las intenciones de Buenos Aires, la vuelta al régimen de la colonia. De entre estos personajes, empezaría a surgir la figura de Juan Manuel de Rosas, que con una extraordinaria habilidad y desde la imagen de caudillo federalista lograría establecer una suerte de gobierno central que se instaló en el actual barrio de Palermo.

Podemos decir que por primera vez en la historia de la ciudad, el escenario protagónico ya no es la plaza. Durante este periodo el eje de la vida política porteña se traslada a las afueras, a la zona de Palermo, que hasta ese momento no estaba incluida dentro del área de la ciudad. Este es el punto de partida de un proceso lento de descompresión de ciertas actividades que resulta muy interesante analizar ya que es indicador de los cambios de modelo institucional, de escala urbana, etc.

Por eso se puede leer como una jugada muy astuta de Rosas, que la sede del gobierno se emplazara en el caserón de Palermo, ya que aunque éste estaba en Buenos Aires, no era la casa de gobierno dentro del fuerte, que se asociaría fácilmente a la propuesta rivadaviana opuesta a la imagen que éste quería dar.

A mediados del siglo XIX comenzaban a llegar a estas tierras las tendencias italianizantes y los caudillos dieron cuenta de esto construyendo importantes residencias donde predominaban los arcos de medio punto, las pilastras y entablamentos clásicos articulando las fachadas. El Palacio San José (1848-1854) obra del arquitecto italiano Pedro Fossati, es uno de los mejores exponentes, junto con la residencia de Rosas ya mencionada. Si bien esta tendencia en el interior del país resultaba innovadora y de vanguardia, 
la ciudad de Buenos Aires ya desde el siglo XVIII contaba con ejemplos de esta arquitectura por su condición de puerto, y sitio de entrada de artistas y constructores desde Europa.

La Plaza de Mayo estuvo hasta la caída del gobierno rosista en una suerte de abandono respecto de la obra realizada; solo las intervenciones particulares dieron cierta renovación al paisaje. Esta situación cambiaria significativamente en el año 1852, en el que los hombres de la generación del 37 llegan al poder para continuar por un lado la unificación del país lograda por el caudillo, pero con las ideas políticas y sociales principalmente francesas sobre las que se inicia el proceso de formación del estado constitucional. Las guerras quedan en cierto modo en el pasado, y Buenos Aires vuelve a ser la capital de la Argentina; esto genera un crecimiento económico y la necesidad de dotar a la ciudad de la infraestructura necesaria para cumplir con la función que lentamente adquiere, y para renovar la edificación obsoleta.

\section{Referencias bibliográficas}

ABÓS, A. (compilador) (2000) El libro de Buenos Aires “Crónicas de cinco siglos”. Buenos Aires: Mondadori.

Anónimo (1937) Boletín de Obras Públicas, Ministerio de Obras Públicas de la república Argentina. (36), pp. 1096.

BORGES, J. L. (1925) Luna de Enfrente. Buenos Aires: Editorial Proa.

CHRISTOPHERSEN, A. (1924) Semejanza de la Música y de la Arquitectura. Revista de la Sociedad Central de Arquitectos, (23), 224-231.

DNA (1934) Intervenciones en la Plaza de Mayo. Revista de la Sociedad Central de Arquitectos, (167), pp. 486.

LEVISMAN, M y CUNIOLO, M. (1989) Alejandro Bustillo Arquitecto 1889-1982. Córdoba: Centro de Arte Contemporáneo.

MUJíCA LÁINEZ, M. (1950) Misteriosa Buenos Aires. Buenos Aires: Editorial Sudamericana.

NOEL, M. (1946) La Trayectoria Puneña y el Barroco Jesuítico, Documentos de Arte Argentino, Cuaderno XIV. Buenos Aires: Academia Nacional de Bellas Artes.

OBLIGADO, P. (1903. Tradiciones Argentinas. Barcelona: Montaner y Simon Editora.

ROMERO, J. L. (1956) Las ideas políticas en Argentina. Buenos Aires: Fondo de Cultura Económica.

SOLÁ, M. (1935) Historia del Arte Hispano-Americano. Barcelona: Editorial Labor.

VITRUVIO, M. L. (1997) Los Diez Libros de Arquitectura. Barcelona: Editorial Iberia.

Recibido: 7 de mayo de 2019

Aceptado: 29 de octubre de 2019 